\title{
Research on Calculation Algorithms of Sea Target Velocity and Course for Sea Radar
}

\author{
Xiaobin Lin ${ }^{1,2, a}$, Chengzhi Zhang ${ }^{1,2}$, Meng Xie ${ }^{1,2}$ and Nan Chen ${ }^{1,2}$ \\ ${ }^{1}$ No. 38 Research Institute of CETC, Hefei 230088, China \\ ${ }^{2}$ Key Laboratory of Aperture Array and Space Application, Hefei 230088, China
}

\begin{abstract}
Sea Radar is an important technical measure for protecting national maritime resources and island sovereignty. The calculation of sea target velocity and course is important to monitor and track sea targets for sea radar, for calculation precision is directly related to the tracking effect of sea target. This paper systematically studies the problem on calculation of sea target velocity and course for sea radar, explicates the relevant technical principles, proposes effective algorithms for calculating sea target velocity and course based on multi-frame accumulative information, and gives a corresponding solution suitable for the scenario of sea target maneuvering. Finally, based on comparison analysis of real radar data and Automatic Identification System (AIS) data, we verify the precision of our algorithms.
\end{abstract}

\section{Introduction}

Sea area monitoring is an important measure to protect national maritime resources and island sovereignty. Sea radar can play a pivotal role in maritime monitoring activities, since it can monitor sea targets at all times and over long distances. Recently, some remarkable works have been made in the research on sea radar technology. Blunt et al. [1] design a heuristic approach, which also performs well when applied to real measured sea clutter for slow target detection. Sira et al. [2] propose a waveform agility based method to improve the detection of sea-surface targets with low RCS. Lei et al. [3] provide a new HF sky-wave radar data processing method TSTM using the CFAR technique to reduce the sea clutter and lessen the echo-broadening and doubleimage. Habtemariam et al. [4] propose a new measurement-level fusion algorithm to combine radar and AIS messages, based on JPDA framework. Liu et al. [5] implement a fuzzy correlation method for target tracking and data fusion to improve the performance and the stability of vessel traffic service.

However, in the process of tracking sea target in sea radar, the precision of sea target velocity and course calculation directly relates to the accuracy of target movement trend prediction and simultaneously affects the filtering processing of target range, target azimuth and other combat indicators. If the velocity and course of sea target is not accurately calculated, it will result in a large deviation between the target's prediction position and its real detection position, causing the target tracking process to be unstable or to lose its follow-up. Therefore, the precision of the calculation of the velocity and course during the tracking of sea target plays a decisive role. Yuan et al. [6] have studied and introduced the relevant concepts of sea target velocity and course solution, real value acquisition and evaluation methods, but there is a certain gap from the actual engineering application. From the perspective of engineering applications for sea target tracking in sea radar, this paper studies the velocity and course calculation algorithms even suitable for sea target maneuvering scenario and utilizes radar and AIS equipment to collect data for comparison analysis to verify the corresponding velocity and course calculation precision of our proposed algorithms.

\section{Calculation Model}

Generally, the radar target measurement parameters are expressed as range, azimuth and height in a polar coordinate system. The height parameter is meaningless for sea target and it is set 0 by default. The radar target tracking effect in the polar coordinate system is shown in Figure 1. The target track number is denoted by $T, j$ represents the $j$-th frame of the target track (the radar is referred to as one frame per scanning cycle period), and the subscripts $p, o$ and $k$ represent the prediction value, the observation value and the filtering value respectively. Since there are noises in the radar system, there are certain errors in the target parameters such as range, azimuth and height. Therefore, the radar data processing software needs to process the target observation parameters by a filtering model. The commonly used filtering models are Kalman filtering model and $\alpha \sim \beta$ filtering model etc. The radar data processing software needs to perform the filtering process on the target observation parameters in the rectangular coordinate system also shown in Figure 1. The relationship among the observation position $\left(x_{o}(T, j), y_{o}(T, j)\right)$, the

*Corresponding author: a cetcxblin@ $163 . c o m$ 
observation range $R_{o}(T, j)$ and the observation azimuth $A_{o}(T, j)$ of the $j$-th frame of the target track $T$ is expressed as Formula 1-2.

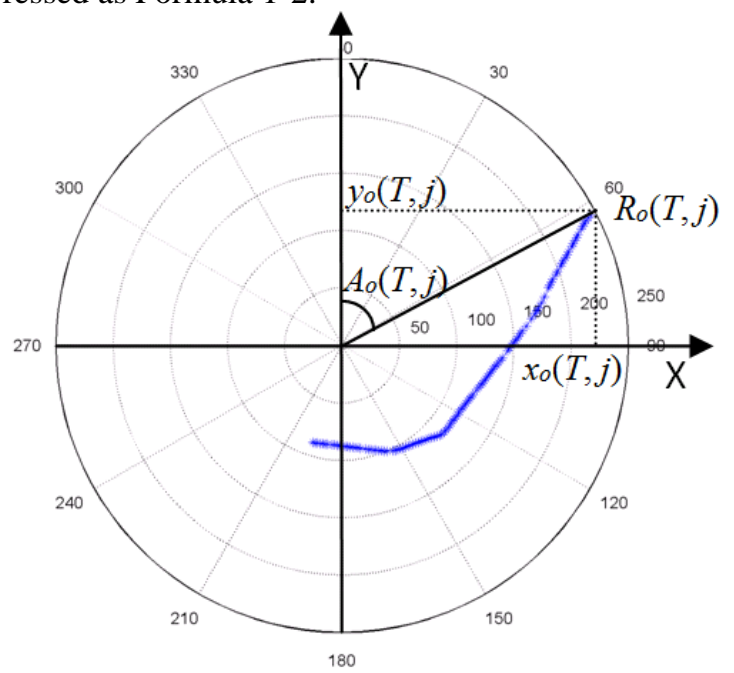

Figure 1. Radar Polar and Rectangular Coordinate System.

$$
\begin{gathered}
x_{o}(T, j)=R_{o}(T, j) \times \sin \left(A_{o}(T, j)\right) \\
y_{o}(T, j)=R_{o}(T, j) \times \cos \left(A_{o}(T, j)\right)
\end{gathered}
$$

In this paper, the filtering position $\left(x_{k}(T, j), y_{k}(T, j)\right)$ of the $j$-th frame of the target track $T$ is obtained based on the observation position $\left(x_{o}(T, j), y_{o}(T, j)\right)$ and the prediction position $\left(x_{p}(T, j), y_{p}(T, j)\right)$, using the $\alpha \sim \beta$ filtering model, as described in Formula 3-4, where $\lambda$ is the filtering gain of the $j$-th frame of the target track $T . \lambda$ is adjusted in time according to the tracking quality of the target track. The value of $\lambda$ is in the range of 0.350.85 in this paper.

$$
\begin{aligned}
& x_{k}(T, j)=x_{p}(T, j)+\lambda \times\left(x_{o}(T, j)-x_{p}(T, j)\right) \\
& y_{k}(T, j)=y_{p}(T, j)+\lambda \times\left(y_{o}(T, j)-y_{p}(T, j)\right)
\end{aligned}
$$

In a general sea radar, the scanning cycle period is 10 seconds (one frame per 10 seconds) and the range detection precision is in the range of 100-meter. The sea target velocity is usually moving slowly at the velocity from 0 to 20 knots, with each knot representing 1.852 $\mathrm{km} / \mathrm{h}$. It means that the normal velocity of sea target is in the range of 0 to $10.3 \mathrm{~m} / \mathrm{s}$. If we calculate the velocity and course of sea target only from the position change of the target in one radar scan cycle period, it will lead huge calculation error, which cannot satisfy the need of the sea target position prediction and filter processing. Therefore, it is necessary to calculate the velocity and course of sea target by multi-frame accumulative position change information to obtain the average position change between multiple frames of sea target.

\subsection{Velocity Calculation Algorithm}

The velocity of the $j$-th frame of the target track $T$ is calculated based on the entire track position change of the previous $N_{v}$ frames. The average position change $\Delta X_{T}^{j}\left(N_{v}\right)$ and $\Delta Y_{T}^{j}\left(N_{v}\right)$ of the target track $T$ from the $j$ th frame to the $\left(j-N_{v}\right)$-th frame on $\mathrm{X}$-axis and $\mathrm{Y}$-axis in the XOY rectangular coordinate system is expressed by Formula 5 and 6 respectively, where SCAN represents the radar scanning cycle period.

$$
\begin{aligned}
\Delta X_{T}^{j}\left(N_{v}\right) & =\frac{x_{k}(T, j)-x_{k}\left(T, j-N_{v}\right)}{N_{v} \times S C A N} \\
\Delta Y_{T}^{j}\left(N_{v}\right) & =\frac{y_{k}(T, j)-y_{k}\left(T, j-N_{v}\right)}{N_{v} \times S C A N}
\end{aligned}
$$

Thus, the velocity of the $j$-th frame of the target track $T$ is calculated as shown in formula 7 . In this paper, $N_{v}$ is in the range from 5 frames to 30 frames.

$$
V_{k}(T, j)=\sqrt{\Delta X_{T}^{j}\left(N_{v}\right)^{2}+\Delta Y_{T}^{j}\left(N_{v}\right)^{2}}
$$

\subsection{Course Calculation Algorithm}

The course of the $j$-th frame of the target track $T$ is calculated by using the entire track position change of the previous $N_{c}$ frames of the target track $T$. The temporary course parameter $C_{k}^{1}(T, j)$ is calculated from the filtering position of the $j$-th frame and the $\left(j-N_{c}+3\right)$-th frame, as shown in Formula 8. The temporary course parameter $C_{k}^{i}(T, j)$ is calculated from the filtering position of the $(j-i)$-th frame and the $\left(j-\left(N_{c}-i+1\right)\right)$-th frame, where $1<i \leq 4$, as shown in Formula 9.

$$
\begin{aligned}
& C_{k}^{1}(T, j)= f\left(x_{k}(T, j)-x_{k}\left(T, j-N_{c}+3\right),\right. \\
&\left.y_{k}(T, j)-y_{k}\left(T, j-N_{c}+3\right)\right) \\
& C_{k}^{i}(T, j)=f\left(x_{k}(T, j-i)-x_{k}\left(T, j-\left(N_{c}-i+1\right)\right),\right. \\
&\left.y_{k}(T, j-i)-y_{k}\left(T, j-\left(N_{c}-i+1\right)\right)\right)
\end{aligned}
$$

The function $f(a, b)$ in Formula 8 and 9 is described as Formula 10.

$$
f(a, b)= \begin{cases}\arctan \frac{a}{b} & \arctan \frac{a}{b} \geq 0 \\ 2 \pi+\arctan \frac{a}{b} & \arctan \frac{a}{b}<0\end{cases}
$$

Based on the temporary course parameter $C_{k}^{i}(T, j)$ calculated by Formula 8 and 9 , where $1 \leq i \leq 4$, we use the four-point smoothing algorithm to calculate the course $C_{k}(T, j)$ of the $j$-th frame of the target track $T$, as shown in Formula 11, where $\rho_{1}, \rho_{2}, \rho_{3}$ and $\rho_{4}$ represent weight coefficients. In this paper, $N_{c}$ is in the range from 5 frames to 30 frames and we set $\rho_{1}=0.35, \rho_{2}=0.25$, $\rho_{3}=0.20$ and $\rho_{4}=0.20$.

$$
C_{k}(T, j)=\sum_{i=1}^{4} \rho_{i} \times C_{k}^{i}(T, j)
$$




\subsection{Target Maneuvering Judgment Algorithm}

When the sea target is maneuvering, the algorithms using the average position change of the multi-frame accumulative information of sea target to calculate the velocity and course are likely to cause large error, if the number of accumulative frames is too large. It may take a long time for the calculated velocity and course of the maneuvering sea target converging to the real value. Therefore, a sea target maneuvering judgment algorithm is proposed to adjust the number of accumulative frames $N_{v}$ and $N_{c}$ for the calculation of velocity and course in time when the sea target maneuvers.

Let $S(T, j)$ be the navigational state of the $j$-th frame of the target track $T$, as shown in Formula 12, where $S(T, j)=1$ demonstrates that the sea target is going to the radar station and $S(T, j)=-1$ demonstrates that the target is leaving from the radar station.

$$
S(T, j)= \begin{cases}1 & R_{o}(T, j)<R_{k}\left(T, j-N_{m}\right) \\ -1 & R_{o}(T, j) \geq R_{k}\left(T, j-N_{m}\right)\end{cases}
$$

In Formula $12, N_{m}$ represents the number of frames needed for judging whether the sea target is maneuvering or not. In this paper, we set $N_{m}=5$. Besides, $R_{k}\left(T, j-N_{m}\right)$ is the filtering range of the $\left(j-N_{m}\right)$-th frame of the target track $T$, as shown in Formula 13.

$$
\begin{aligned}
& R_{k}\left(T, j-N_{m}\right) \\
& =\sqrt{x_{k}^{2}\left(T, j-N_{m}\right)+y_{k}^{2}\left(T, j-N_{m}\right)}
\end{aligned}
$$

Besides, in Formula 12, when $S(T, j)$ is different from $S(T, j-1)$, it indicates that the target track $T$ changes the navigation direction in the $j$-th frame, i.e., the target $T$ is in the process of maneuvering and thus the corresponding number of accumulative frames $N_{v}$ and $N_{c}$ for the velocity and course calculation should be timely adjusted to the minimum value. In this paper, when the sea target maneuvers, we adjust $N_{v}$ and $N_{c}$ to the minimum value of 5 frames.

\section{Performance Evaluation}

Based on the real tracking data of sea target recorded by radar data processing software running in the radar equipment, we carry out the statistical analysis of the precision of the velocity and course calculation algorithms proposed in this paper. The automatic response data from the AIS equipment equipped to the radar is considered as the real value. Thus, we compare the velocity and course data of the proposed algorithms implemented in the radar data processing software with the automatic response velocity and course data obtained from the AIS equipment for the same target to verify the effectiveness of our algorithms.

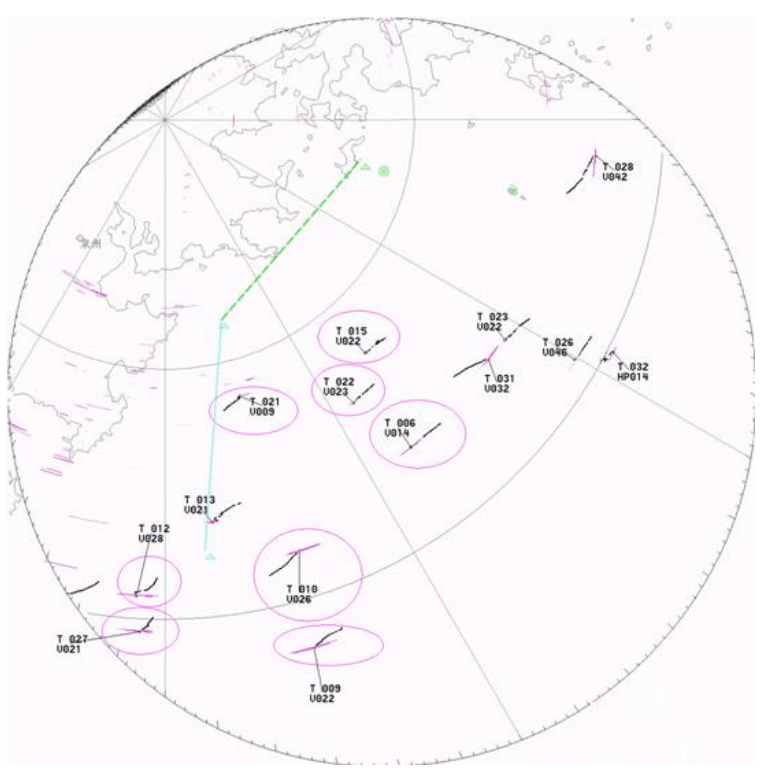

Figure 2. Radar Tracking Screen of Sea Targets.

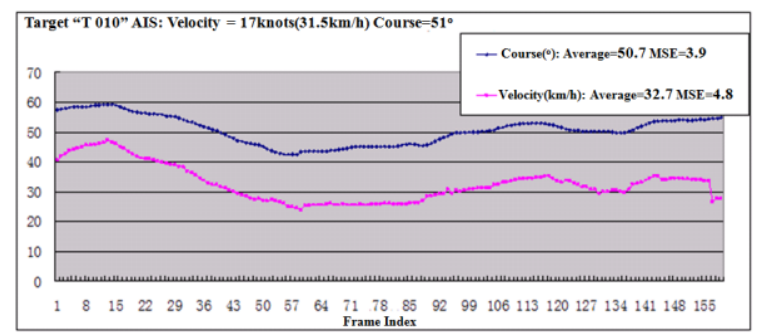

Figure 3. Statistical Results of the Target Track "T 010".

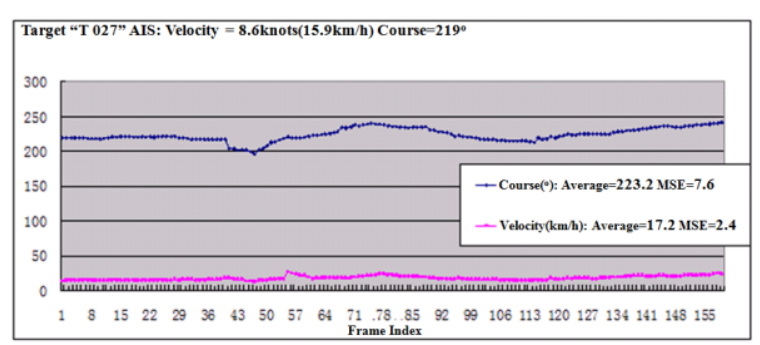

Figure 4. Statistical Results of the Target Track "T 027".

Figure 2 shows the tracking screen of the sea targets by the sea radar. We can see that the tracking of the sea targets is continuous and their routes are smooth. The statistical results of the velocity and course calculation error between the radar target track "T 010" and the corresponding AIS target is shown in Figure 3, where the mean square error (MSE) of the velocity is $4.8 \mathrm{~km} / \mathrm{h}$ and the MSE of the course is 3.9 degrees. The statistical results of the velocity and course calculation error between the radar target track "T 027" and the corresponding AIS target is shown in Figure 4, where the MSE of the velocity is $2.4 \mathrm{~km} / \mathrm{h}$ and the MSE of the course is 7.6 degrees. The results indicate that our algorithms are effective and suitable for sea radars with the following required tracking precision of sea target: velocity calculation error $\leqslant 5$ knots $(9.26 \mathrm{~km} / \mathrm{h})$, course calculation error $\leqslant 10$ degrees.

The sea target maneuvering judgment algorithm proposed in this paper can quickly and accurately identify the maneuvering process of sea target and thus can adjust the number of accumulative frames for the 
calculation algorithms of the velocity and course in time, so that the calculated value of the velocity and course can quickly approximate to the real value, which is beneficial to the prediction and tracking of sea target. The sea target track "T 049" is still stable and smooth when it is maneuvering, as shown in Figure 5, which demonstrates that the proposed sea target maneuvering judgment algorithm is effective for adjusting the number of accumulative frames for our calculation algorithms of the velocity and course in time.

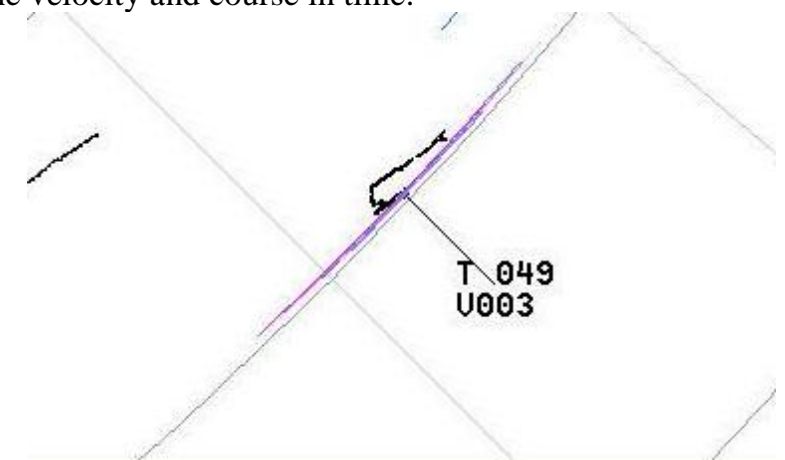

Figure 5. Tracking Effect of the Maneuvering Target "T 049".

\section{Conclusion}

The precision of the velocity and course calculation is directly related to the tracking of sea target, which furthermore affects the precision of the range and azimuth detection. Since the general range detection precision of sea radar is in the 100-meter range and meanwhile the slow sea target cannot move over one range resolution unit of the sea radar, it means that using one-frame position change to calculate the velocity and course is no longer suitable for the slowly moving sea target. This paper systematically studies the problem of sea target velocity and course calculation for sea radar, proposes calculation algorithms of the velocity and course for sea target based on multi-frame accumulative position change, furthermore designs a maneuvering judgment algorithm to adjust the number of accumulative frames for the velocity and course calculation when the sea target is maneuvering and finally based on comparison analysis of the real radar data and AIS data, we verify the precision of our proposed algorithms. The experimental results show that our algorithms can effectively satisfy the velocity and course calculation precision of the general sea radars and can easily be applicable to radar engineering projects with perfect tracking performance.

\section{References}

1. S. D. Blunt, K. Gerlach, J. Heyer, HRR Detector for Slow-Moving Targets in Sea Clutter, IEEE Transactions on Aerospace \& Electronic Systems, vol. 43, no. 3, pp. 965-974(2007).

2. S. P. Sira, D. Cochran, A. Papandreou-Suppappola, et al., Adaptive Waveform Design for Improved Detection of Low-RCS Targets in Heavy Sea Clutter,
IEEE Journal of Selected Topics in Signal Processing, vol. 1, no. 1, pp. 56-66(2007).

3. Q. Lei, Z. Wu, L. Guo, et al., The big data processing of HF sky-wave radar sea echo for detection of sea moving targets, International Journal of Information Technology \& Web Engineering, vol. 12, no. 4, pp. 56-71( 2017).

4. B. Habtemariam, R. Tharmarasa, M. Mcdonald, et al., Measurement level AIS/radar fusion, Signal Processing, vol. 106, pp. 348-357(2015).

5. C. Liu, M. Cao, F. Han, et al., A model for fuzzy data correalation of ais and radar, International Journal on Smart Sensing \& Intelligent Systems, vol. 5, no. 4, pp. 843-858(2012).

6. G. Yuan, A study on calculating and validation of target course and speed, Radar \& Ecm, no. 1, pp. 26-28(2009). 